\title{
"Estimation of Serum Amyloid A, CRP, Homocysteine, and Neutrophils to Lymphocyte Ratio as an Inflammatory biomarker and their correlation with Cardiovascular risk in a sample of Iraqi COVID-19 Patients"
}

\author{
*Hayder Y. Ali \\ ** Dr.Mohammed Abdulateef Al-bayati \\ *** Dr. Haider Abdulhameed Alqaraghuli \\ */ Al-Nahrain University /College of Medicine / Department of Chemistry and Biochemistry / Iraq \\ **/Al-Nahrain University /College of Medicine / Department of Chemistry and Biochemistry / Iraq \\ ***/ Al-Nahrain University /College of Medicine / Department of Medicine / Iraq \\ E.mail: haydar.yaseen.ali@gmail.com \\ Mobile : +9647711327082
}

\begin{abstract}
BACKGROUND: COVID-19 is a disease caused by a new coronavirus called SARS CoV-2. WHO first learned of this new virus on 31 December 2019, following a report of a cluster of cases of 'viral pneumonia in Wuhan, the People's Republic of China.

AIM: The study aims to explore the clinical values of combined detection of serum concentration of SAA, C reactive protein, Neutrophil-Lymphocyte ratio, D-dimer, and homocysteine in SARS-COV-2 infected patients, in hospitalized patients, with reference to other inflammatory, metabolic parameters, as well as cardiovascular risk factors and if it can also assess in the monitoring the efficiency of COVID- 19 treatment.

METHOD: A cross-sectional study was employed. It was conducted in multi-hospital of Baghdad city. All patients in this study were hospitalized patients with PCR confirmed. targeted population included 42 patients (male \& female) aged (18 - 80 years). patients were divided into two groups according to the outcomes of Survival 19 patients and non-survival 23 patients. All biochemical parameters were measured on survivors and non-survivors groups at two different time points.

RESULTS: The difference of comparison of SAA and other biomarkers between survival group and nonsurvival group at two different time points hospital admission T1 and after 7 days from admission and starting therapy T2, was statistically significant. The ROC curve results showed that SAA has higher sensitivity to the predictive value of 7 days after treatment.

CONCLUSION: SAA high sensitive indicators in estimating the severity and prognosis of COVID-19. And can be used for monitoring the recovery process in COVID-19 patients. SAA was more efficient in predicting COVID-19 than other markers.

KEYWORDS: Iraqi COVID-19, Serum Amyloid A, Homocysteine and Cardiovascular risk.
\end{abstract}

\section{Introduction:}

Coronaviruses are enveloped, single-stranded, positive RNA viruses that infect humans and other animals $(1,2)$. These virions are spherical, with a core-shell structure and a surface that resembles a solar corona. Thus, they are known as coronaviruses (corona is Latin for crown) (3). COVID-19 is a disease caused by a new coronavirus called SARS-CoV-2.WHO first learned of this new virus on 31 December 2019, following a report of a cluster of cases of "viral pneumonia in Wuhan, the People's Republic of China (4). Most people infected with the SARSCoV-2 will have a respiratory illness, which is usually mild to moderate. They will 
be fine without medical treatment. The likelihood of getting serious illnesses increases with age and chronic illnesses like cardiovascular disease, diabetes, chronic respiratory disease, and cancer. Breathing may become more difficult due to the lungs becoming inflamed. This results in critical care needs for some individuals $(4,5)$. Knowing the role that viral pathogens play in causing disease and the type of cell and organ damage caused is an essential part of finding new biomarkers that can help identify and diagnose viral pathogenetic pathways. An effective and reliable biomarker for use in screening, clinical care, and the prevention of significant consequences would be very beneficial and differentiate the severity of COVID-19 and make improvements to reduce mortality for patients infected with it (6).

Serum amyloid A (SAA) proteins were isolated and named over 50 years ago. They are small (104 amino acids) and have a special relationship to the acute phase response, with serum levels rising 1000-fold in 24 hours (7). The precursor of amyloid is found in SAA, a plasma component. Proinflammatory cytokines generated by active monocytes are responsible for liver production of SAA, an acute-phase protein; SAA rises dramatically in response to acute Inflammation and damage, reaching 1000-fold over normal levels within 24 hours. SAA plays a vital role in Inflammation and associates with the severity of Inflammation $(8,9)$.

The kinetics of SAA induction appears to be sensitive to the cytokine that promotes the liver to produce SAA during Inflammation, which is most frequently found to be IL-1, IL-6, and TNF- $\alpha$. Additionally, cytokines like IL-2 and interferon- $\gamma$ (IFN- $\gamma)$ may influence A-SAA induction. As long as the other cytokine was present in small quantities, raising the SAA production amount significantly increased with rising concentrations of the one cytokine $(10,11)$.

The acute phase response results in an increased plasma SAA protein level, which causes HDL remodeling. HDL remodeling includes the production of new SAA1 and SAA2 that displace apoA-1 and become an apolipoprotein of HDL. Nevertheless, apoA-1 cannot replace SAA in HDL molecule formation; the role of SAA in lipid metabolism is widely important, but how SAA affects lipid metabolism is poorly understood $(12,13)$. The accumulation of evidence indicates that SAA, which is present during the early stages of Inflammation, is an active particle in Inflammation. Immunologically active, cytokine-like characteristics and several proinflammatory activities are found in SAA. In cellular response to SAA, the concentration of SAA is a determining factor for both cell type and location. Tumor necrosis factor- $\alpha$ (TNF- $\alpha$ ), interleukin-1 $\beta$ (IL-1 $\beta$ ), and interleukin-8 (IL-8) are all created by human neutrophils and mast cells in response to SAA. 


\section{Materials and Methods:}

A cross-sectional study was employed. It was conducted in multi-hospital of Baghdad city. The study was approved by the scientific committee "Institutional Review Board" (IRB) in Al-Nahrain University, College of Medicine. All patients in this study were hospitalized patients with PCR confirmed SARS-COV2 (severe cases, critical cases) according to Iraqi guidelines and supervision of a specialist. The targeted population included 43 patients of both genders (male \& female) aged (18 - 80 years). Patients who were followed treated according to the guidelines of Iraqi $\mathrm{MOH}$, of conventional treatment including early effective oxygen therapy, glucocorticoids, and antiviral and nutritional support, all patients were followed up for (two months). one patient was excluded due to loss of follow-up. The remainder of 42 patients were divided into two groups according to the outcomes, Survival 19 patients and non-survival 23 patients. All biochemical parameters were measured on survivors and non-survivors groups at two different time points hospital admission T1 after seven days from admission and starting therapy T2.

Inclusion criteria

A) severe disease cases

those who meet one of the following criteria for the diagnosis of severe cases according to the Iraqi MOH guide:

(1) respiratory distress, $R R \geq 30$ breaths $/ \mathrm{min}$.

(2) pulse oxygen saturation $(\mathrm{SpO} 2) \leq 93 \%$ on room air at rest state.

(3) patients with $>50 \%$ lesions progression within $24-48 \mathrm{~h}$ in pulmonary imaging.

B) critically diseased cases

those who meet one of the following criteria are diagnosed as critically ill cases:

(1) respiratory failure and need for mechanical ventilation.

(2) shock.

(3) complicated with other organ failures that require monitoring and treatment. Exclusion criteria

(1) age $<18$ years. (2) pregnant or lactating women. (3) treatment period less than seven days. (4) patients with a history of advanced liver or chronic kidney diseases, advanced malignant tumors, and other serious underlying diseases. (5) any loss of contact with already enrolled patients during follow-up.

Fasting venous blood was taken from all 43 hospitalized patients into a proper tube for whole blood and serum separation, then subsequent measurement of the following parameters Serum Amyloid A (SAA), Homocysteine, C-reactive protein (CRP), D-dimer, lipid profile, and Complete blood count (CBC).

The medical and social history was taken from each patient according to the questionnaire. All patients provided informed consent to participate in the study, data collection, and analysis for research purposes and were asked to answer a 
prepared questionnaire after their consent had been obtained by direct interview with them.

Blood pressure (BP) was assessed in a sitting position. Other clinical ad demographic parameters were measured, and Some were taken from patients' hospital records.

ROC curve was utilized to analyze the sensitivity of SAA, CRP, Homocysteine, and NLR of prognosis of patients with COVID-19. The SAA and Hcy levels were measured via ELISA (Enzyme-Linked Immunosorbent Assay) Kit, using Automatic Elisa Reader and washer Biotek. Lipid profile was measured by Roche Diagnostic Cobas C311 analyzer. D-Dimer and CRP were measured via fluorescence Immunoassay (FIA) by AFIAS-6.

The data were analyzed using Statistical Package for Social Sciences (SPSS) version 25. Statistical data including frequency, mean and standard deviation were measured to describe the variables. The groups were compared by applying an independent sample t-test (unpaired t-test between two groups) and repeated measure ANOVA (evaluate interactions between patients' groups and Time of measurements).

\section{Results:}

Clinical characteristics of patients with COVID-19 at admission. within the 42 patients, $24(57.1 \%)$ were men, and $18(42.9 \%)$ were women, including (severe and critical) cases who were hospitalized with laboratory-confirmed COVID-19. patients categorized into $19(45.2 \%)$ survival and $23(54.8 \%)$ non-survival groups. The mean age was $(60.30 \pm 13.97)$ years in non-survival patients and $(59.53 \pm$ 9.06) years in survival groups. (Table 1). Compared with the survival group, the non-survival group was significantly being female [mean 13 (56.5\%) female vs. 10 $(43.5 \%)$ male; $\mathrm{P}=0.049]$. Also hypertension [11 (47.8\%) vs. 8 (42.1\%)] and Obesity [7 (36.8\%) vs. 9 (39.1\%)] (Figure 1).

There was also a significant difference in oxygen saturation between survivals and non-survivals with higher levels in the non-survival group $(85.9 \%$ versus $77 \%$ \& $\mathrm{p}=0.001$ ). (Figure 2). 
Table 1. Clinical characteristics of 42 patients with COVID-19 upon admission

\begin{tabular}{|l|l|l|l|l|}
\hline \multicolumn{2}{|l|}{ Parameters } & Survivals & Non-survivals & $p$-value \\
\hline Age (years) & $59.53 \pm 9.06$ & $60.30 \pm 13.97$ & 0.836 \\
\hline Duration of illness (days) & Male & $26.74 \pm 8.56$ & $26.83 \pm 9.06$ & 0.986 \\
\hline \multirow{2}{*}{ Gender } & Female & $5(26.3 \%)$ & $10(43.5 \%)$ & \multirow{2}{*}{$0.049^{*}$} \\
\hline Diabetes history & & $3(15.8 \%)$ & $13(56.5 \%)$ & 0.313 \\
\hline Hypertension history & $8(42.1 \%)$ & $1(4.3 \%)$ & 0.711 \\
\hline Smoking history & $2(10.5 \%)$ & $11(47.8 \%)$ & 0.199 \\
\hline Obesity & $7(36.8 \%)$ & 0 & 0.879 \\
\hline
\end{tabular}

Figure 1: Comparison of demographic features between survivals and non-survivals

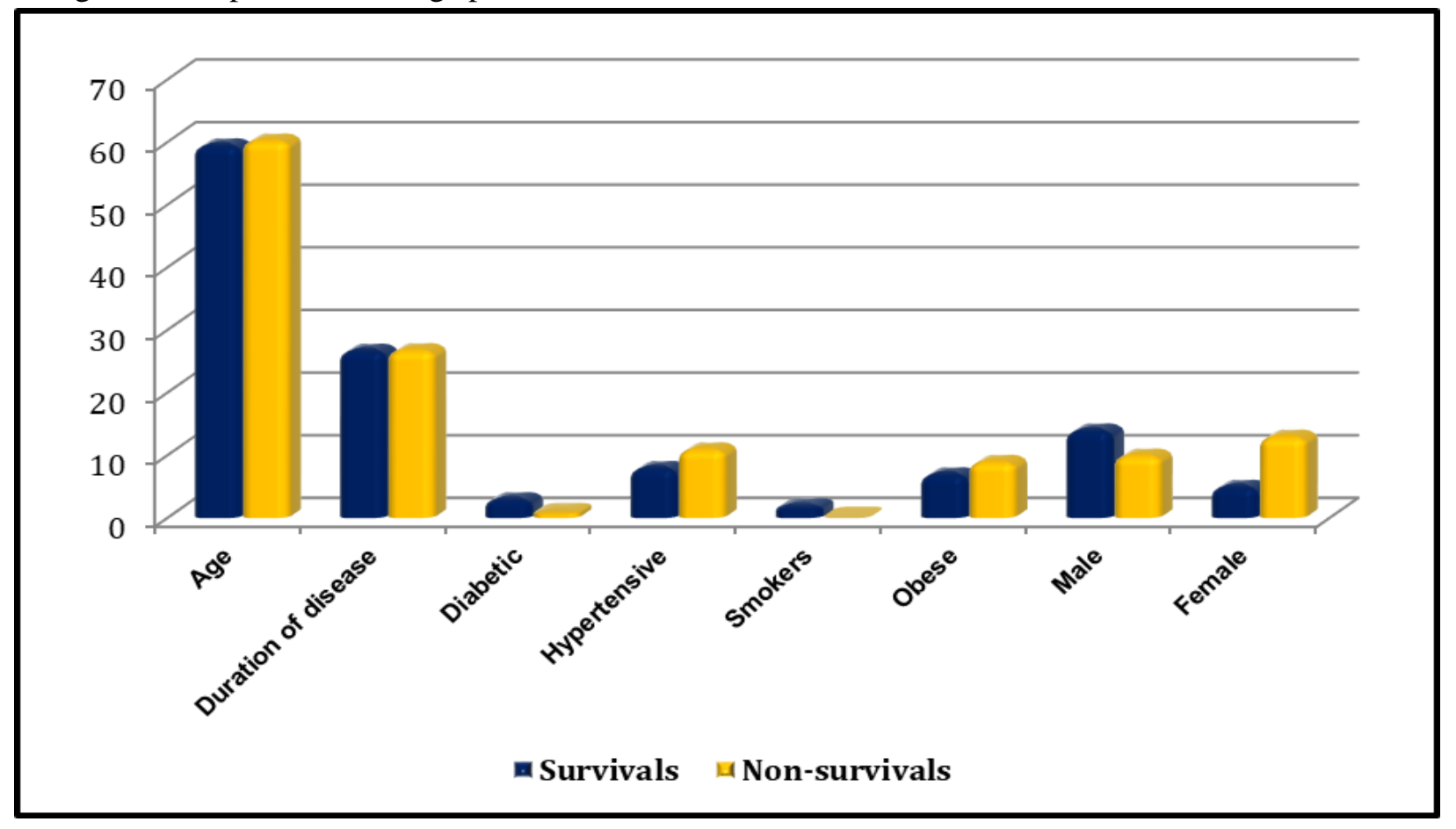




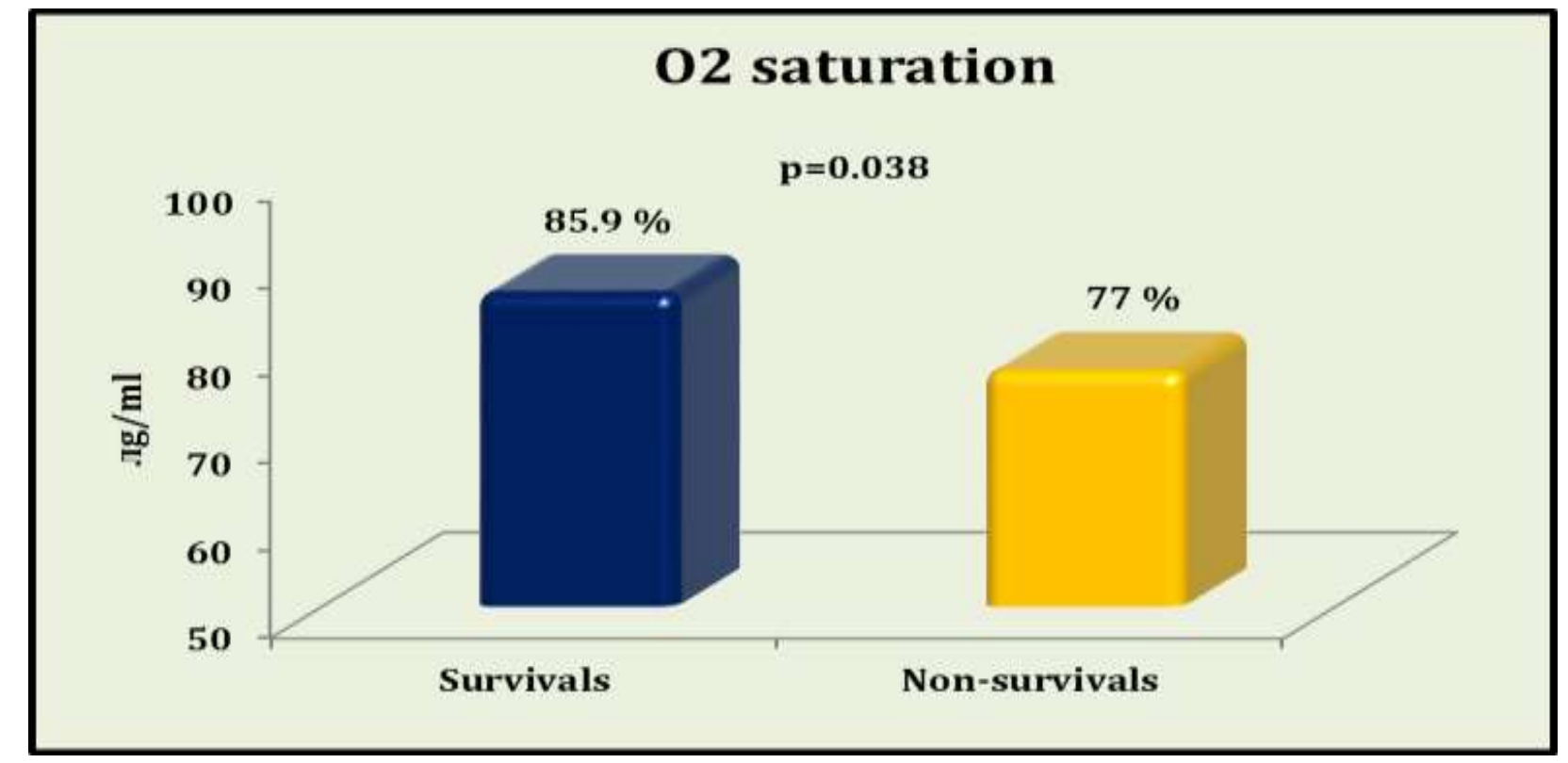

Figure 2: Comparison of $\mathrm{O} 2$ saturation levels between survivals \& non-survivals

Laboratory findings of patients with COVID-19 at admission and after seven days from admission. The present study reported the results of blood routine and inflammation-related biomarkers of the patients with COVID-19 upon hospital admission. The results indicated that Serum amyloid-A, homocysteine, CRP, and neutrophils to lymphocytes ratio (NLR) and D-dimer were significantly different between two different time points hospital admission (T1) and after seven days from admission and starting therapy (T2), at the surviving group and the nonsurviving group. Were presented in tables ( 2 and 3 ).

This study investigated SAA levels at admission (T1) and after seven days (T2) of hospitalization in two groups of patient survivors and non-survivors.

In both groups, outcomes show significant differences between $\mathrm{T} 1$ and $\mathrm{T} 2$. in nonsurvivors, At T2, showed higher SAA level than the T1 mean value $(132.24 \mathrm{mg} / \mathrm{L}$ vs. $153.78 \mathrm{mg} / \mathrm{L})$ with $(P<0.001 *)$. In survivors, the group showed a decreased level of SAA at T2 than T1, mean value $(140.35 \mathrm{mg} / \mathrm{L}$ vs. $98.84 \mathrm{mg} / \mathrm{L})$ with $(P<$ $0.001 *)$. 
Table 2: Paired samples T-test between inflammatory biomarkers measurements in survivals

\begin{tabular}{|l|l|l|l|}
\hline Inflammatory biomarker & $\begin{array}{l}1^{\text {st }} \text { measurement } \\
(\text { Mean } \pm \text { SD })\end{array}$ & $\begin{array}{l}2^{\text {nd }} \text { measurement } \\
(\text { Mean } \pm \text { SD })\end{array}$ & $p$-value \\
\hline Serum amyloid-A (mg/l) & $140.35 \pm 26.18$ & $98.84 \pm 28.51$ & $<0.001^{*}$ \\
\hline $\begin{array}{l}\text { Serum Homocysteine } \\
(\mathrm{nmol} / \mathrm{ml})\end{array}$ & $23.32 \pm 12.33$ & $21.31 \pm 9.87$ & 0.294 \\
\hline $\begin{array}{l}\text { C-Reactive Protein }(\mathrm{mg} / \mathrm{l}) \\
\text { Neutrophils to lymphocytes } \\
\text { ratio }\end{array}$ & $82.55 \pm 41.88$ & $34.85 \pm 19.13$ & $<0.001^{*}$ \\
\hline $\begin{array}{l}\mathrm{D}-\mathrm{dimer} \\
(\mu \mathrm{g} / \mathrm{ml})\end{array}$ & $1.66 \pm 1.52$ & $8.63 \pm 2.95$ & $<0.001^{*}$ \\
\hline
\end{tabular}

Table 3: Paired samples t-test between inflammatory biomarkers measurements in non-survivals

\begin{tabular}{|l|l|l|l|}
\hline Inflammatory biomarker & $\begin{array}{l}1^{\text {st }} \text { measurement } \\
(\text { Mean } \pm \text { SD })\end{array}$ & $\begin{array}{l}2^{\text {nd }} \text { measurement } \\
(\text { Mean } \pm \text { SD })\end{array}$ & $p$-value \\
\hline$(\mathrm{mg} / \mathrm{l})$ & $132.24 \pm 29.98$ & $153.78 \pm 23.23$ & $<0.001^{*}$ \\
\hline $\begin{array}{l}\text { Serum Homocysteine } \\
(\mathrm{nmol} / \mathrm{ml})\end{array}$ & $20.85 \pm 9.17$ & $27.78 \pm 9.03$ & $0.004^{*}$ \\
\hline $\begin{array}{l}\text { C-Reactive Protein }(\mathrm{mg} / \mathrm{l}) \\
\text { Neutrophils to lymphocytes } \\
\text { ratio }\end{array}$ & $68.47 \pm 38.93$ & $86.07 \pm 52.13$ & $0.026^{*}$ \\
\hline $\begin{array}{l}\mathrm{D}-\mathrm{dimer} \\
(\mu \mathrm{g} / \mathrm{ml})\end{array}$ & $3.26 \pm 2.91$ & $30.13 \pm 18.35$ & $0.047^{*}$ \\
\hline
\end{tabular}

Two way repeated measures ANOVA have been applied for the comparison of inflammatory biomarkers measurements interaction with survivals and nonsurvivals groups, the results were shown significant differences in Serum amyloid- 
A levels, regarding the interaction between patients groups and Time of inflammatory biomarkers readings the results showed interactions with serum amyloid-A and C-reactive protein meaning that there is combined effect for Time and patients groups (survivals and non-survivals ). (table 4) and (figure 3).

Table 4: interactions serum amyloid-A for Time and patients' groups (survivals and non-survivals)

\begin{tabular}{|l|l|l|l|l|l|}
\hline \multicolumn{2}{|l|}{ survival and non-survival * time } \\
\hline Measure: SAA & \multirow{2}{*}{ Time } & \multirow{2}{*}{ Mean } & Std. Error & \multicolumn{2}{l|}{$95 \%$ Confidence Interval } \\
\cline { 4 - 7 } Survival \& non-survival & Timer Bound & Upper Bound \\
\hline \multirow{2}{*}{ non-survival } & 1 & 132.2 & 5.9 & 120.3 & 144.1 \\
\hline \multirow{2}{*}{ survival } & 2 & 153.7 & 5.3 & 142.9 & 164.6 \\
\cline { 2 - 7 } & 1 & 140.3 & 6.5 & 127.2 & 153.4 \\
\cline { 2 - 7 } & 2 & 98.8 & 5.9 & 86.9 & 110.7 \\
\hline
\end{tabular}

Figure 3: Serum Amyloid A level in patients with COVID-19 infection according to survivals and nonsurvivals interactions with Time.

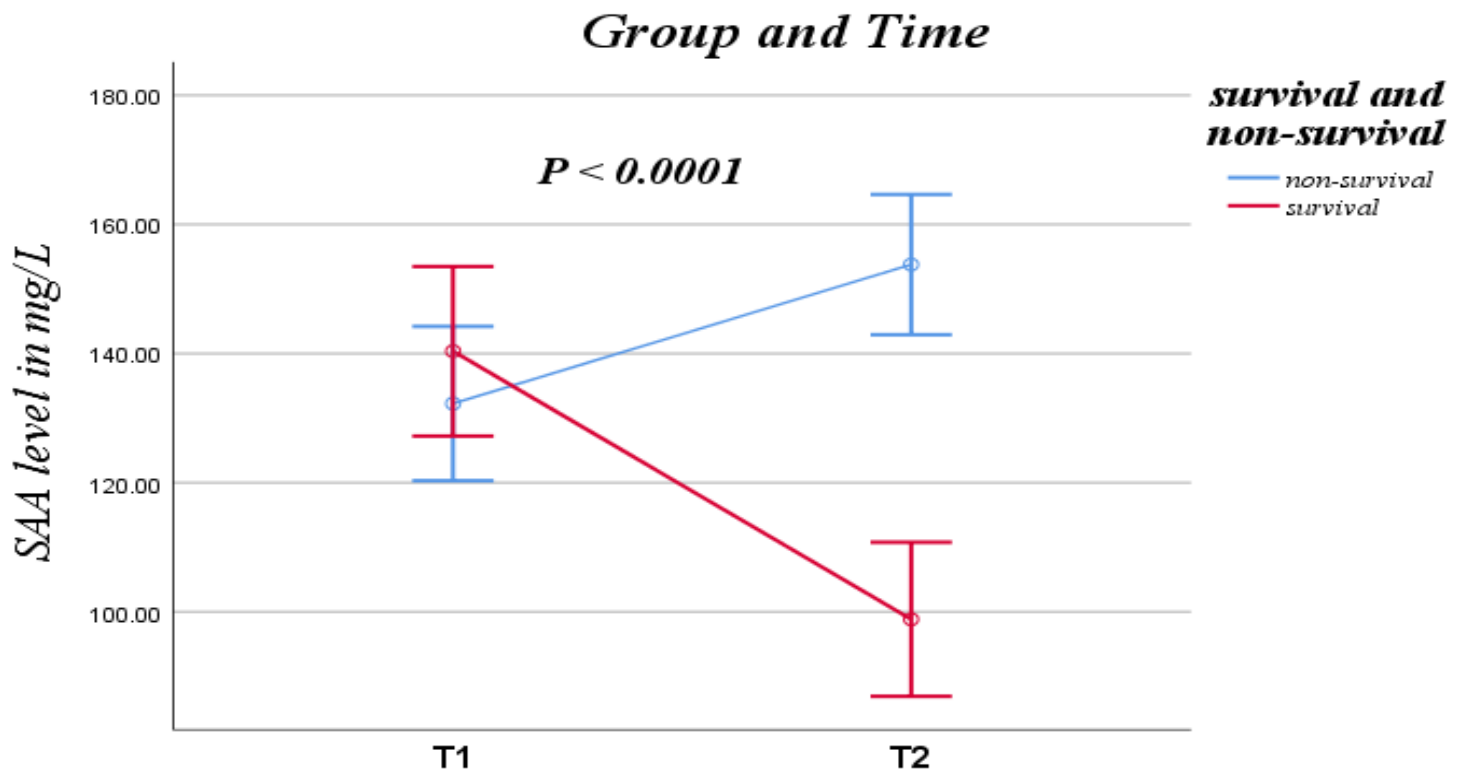

\section{Time}

The ROC curve was used to analyze the early-warning efficiency and the optimal prediction threshold of COVID-19 severity. The corresponding AUC of SAA had the largest area under the curve at values greater than $129.1 \mathrm{mg} / \mathrm{l}(\mathrm{AUC}=0.922)$ with sensitivity and specificity of $87 \%$ and $89.5 \%$, respectively, to recover COVID-19. The present study results indicated that SAA could be considered a 
biomarker for predicting the severity and recovery of COVID-19 in the survival and non-survival group (Figure 4)

SAA levels were negatively correlated with treatment days in the survival group. Dramatically decrease the level of all biomarkers after one week of starting treatment in recover patients (Figure 5).

Figure 4: ROC curve findings of SAA in patients with COVID-19 infection according to survivals and

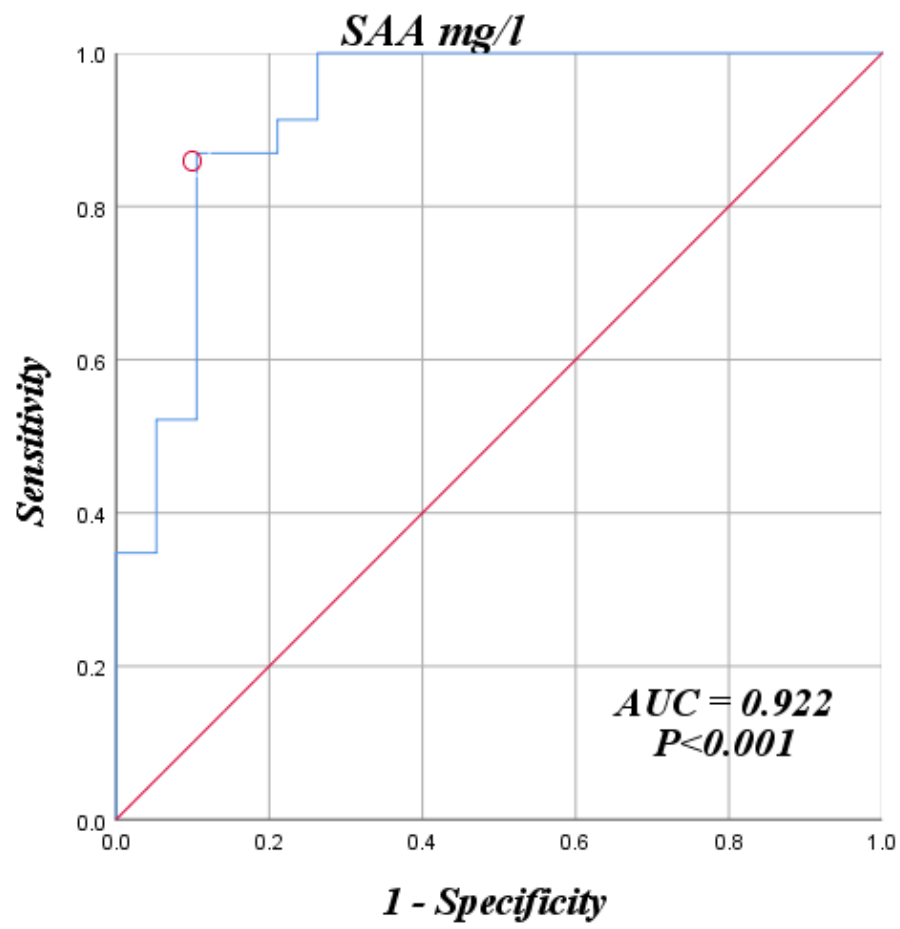

non-survivals. 


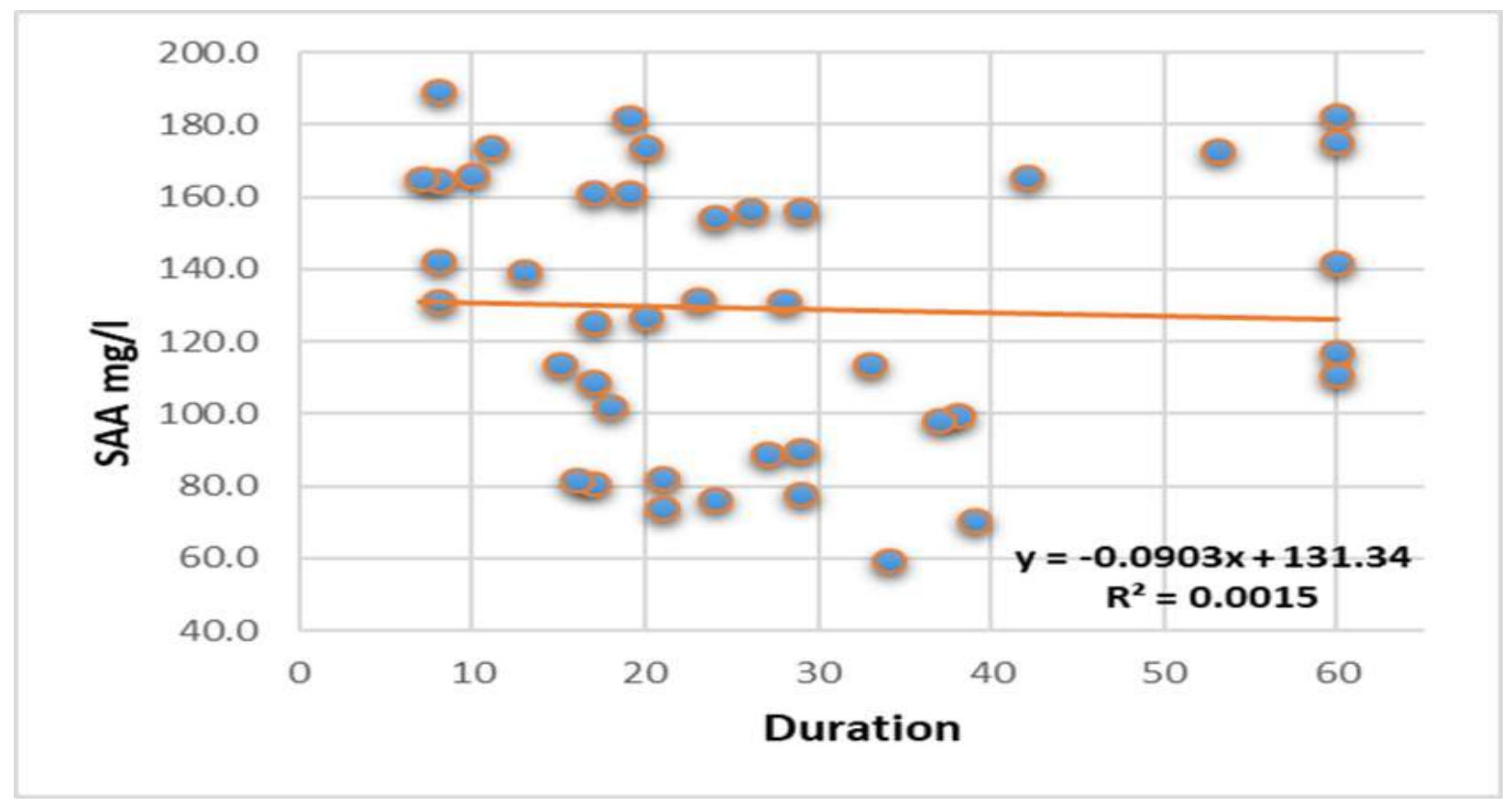

Figure 5: Scatter-plot showing the correlation between SAA and Duration in COVID-19 patients.

\section{Discussion:}

The current study reported a cross-sectional study of 42 patients, 24 (57.1\%) were men and 18 (42.9\%) were women, including (severe and critical) cases who were hospitalized with laboratory-confirmed COVID-19 in Baghdad, Iraq. All patients were followed up for 60 days after the ending of the sampling for survival and prognosis, and to follow up, we contacted all patients by telephone through 60 days. Based on the 60-day follow-up results, we divided 42 cases with COVID-19 into survival groups (19 cases, 45.2\%) and non-survival groups (23 patients $54.8 \%$ ). The end event point for non-survival considered complication with other organ failures that require special care such as heart injury, chronic lung fibrosis, or chronic fatigue or death during the follow-up time.

Within this cross-sectional, the non-surviving patients were the most critical cases of COVID-19; compared with the surviving group, most of them have severe cases. The most common symptoms were fever, cough, Chest tightness, and Shortness of breath. Fever was the first symptom reported by many patients $(88.1 \%)$. Identifying patients with a poor prognosis can serve as an indicator of the onset of dyspnea. This point often helps doctors in diagnosing cases. Approximately $(42.8 \%)$ of patients were identified as critical and (57.1\%) severe at the Time of admission.

We reported the death of 19 patients who were part of the COVID-19 research in this study. Hypoxemia was difficult to recover in most patients due to the presence of pulmonary consolidation. Age and underlying diseases are considered the most 
critical risk factors for death in these patients. The mean age was $(60.30 \pm 13.97)$ years in non-surviving patients. The most common underlying disease was hypertension and, at a lower rate, cardiovascular disease and diabetes. The effect of age and comorbidities has been discussed in other studies $(14,15)$. These studies showed for every five years increase in age, the risk of death increased by 1.55 .

There was also a significant difference in oxygen saturation $(\mathrm{SpO} 2)$ between survivals and non-survivals with higher levels in the non-survival group (85.9\% versus $77 \% \& \mathrm{p}=0.001$ ). These results agree with (16), who showed that patients in severe groups had a lower $\mathrm{SpO} 2$ than patients in non-severe groups.

The study's major finding was that serum SAA concentration was high above normal in the vast majority of COVID-19 patients. The normal SAA level $<10$ $\mathrm{mg} / \mathrm{l}$. Dynamically monitoring the changes in SAA to adopt an effective diagnosis and treatment strategy for COVID-19 patients in the early stage may improve the survival rate of patients.

Conclusions:

SAA, CRP, NLR, D-dimer, and Homocysteine levels were increased in the nonsurvival group and decrease in the survival group after seven days from admission and starting therapy. SAA levels were negatively correlated with treatment days in the survival group. Dramatically decrease the level of all biomarkers after one week of starting treatment in recover patients. SAA high sensitive indicators in estimating the severity and prognosis of COVID-19. And can be used for monitoring the recovery process in COVID-19 patients. SAA was more efficient in predicting COVID-19 than other markers.

\section{Reference:}

1. Velázquez-Silva RI. Historia de las infecciones por coronavirus y epidemiología de la infección por SARS-CoV-2. Rev Mex Traspl. 2020;9(S2):149-59.

2. Velavan TP, Meyer CG. The COVID-19 epidemic [Internet]. Vol. 25, Tropical Medicine and International Health. Blackwell Publishing Ltd; 2020 [cited 2021 Mar 25]. p. 278-80. Available from: /pmc/articles/PMC7169770/

3. Zhou P, Yang XL, Wang XG, Hu B, Zhang L, Zhang W, et al. R.; Zhu, Y.; Li, B.; Huang, C. L, Chen H-D, Chen J, Luo Y, Guo H, Jiang R-D, Liu M-Q, Chen Y, Shen X-R, Wang X, Zheng X-S, Zhao K, Chen Q-J, Deng F, Liu L-L, Yan B, Zhan FX, Wang Y-Y, Xiao G-F, Shi Z-L A pneumonia outbreak Assoc with a new coronavirus . 2020;579:270-3.

4. WHO. Coronavirus (COVID-19) disease,Overview [Internet]. Overview, WHO. 2021 [cited 2021 Apr 4]. Available from: https://www.who.int/healthtopics/coronavirus\#tab=tab_1 
5. Gupta A, Madhavan M V., Sehgal K, Nair N, Mahajan S, Sehrawat TS, et al. Extrapulmonary manifestations of COVID-19. Nat Med. 2020;26(7):1017-32.

6. $\mathrm{Xu} \mathrm{J}, \mathrm{Xu} \mathrm{C}$, Zhang $\mathrm{R}, \mathrm{Wu} \mathrm{M}$, Pan C, Li X, et al. Associations of procalcitonin, $\mathrm{C}$-reaction protein and neutrophil-to-lymphocyte ratio with mortality in hospitalized COVID-19 patients in China. Sci Rep [Internet]. 2020 Dec 14;10(1):15058. Available from: https://doi.org/10.1038/s41598-020 72164-7

7. Sack GH. Serum amyloid A - A review. Vol. 24, Molecular Medicine. BioMed Central Ltd.; 2018.

8. Vietri L, Fui A, Bergantini L, D'Alessandro M, Cameli P, Sestini P, et al. Serum amyloid A: A potential biomarker of lung disorders [Internet]. Vol. 58, Respiratory Investigation. 2020 [cited 2021Apr20].p.21-7.Availablefrom: https://www.sciencedirect.com/science/article/pii/S2212534519301650

9. Kushner I, Mackiewicz A. The acute phase response: an overview. Acute phase proteins Mol Biol Biochem Clin Appl. 1993;3.

10. Thorn CF, Whitehead AS. Differential glucocorticoid enhancement of the cytokine-driven transcriptional activation of the human acute phase serum amyloid A genes, SAA1 and SAA2. J Immunol. 2002;169(1):399-406.

11. De Buck M, Gouwy M, Wang JM, Van Snick J, Proost P, Struyf S, et al. The cytokine-serum amyloid A-chemokine network. Cytokine Growth Factor Rev. 2016;30(2015):55-69.

12. Jahangiri A, de Beer MC, Noffsinger V, Tannock LR, Ramaiah C, Webb $\mathrm{NR}$, et al. HDL remodeling during the acute phase response. Arterioscler Thromb Vasc Biol. 2009;29(2):261-7.

13. Webb NR. High-Density Lipoproteins and Serum Amyloid A (SAA) [Internet]. Vol. 23, Current atherosclerosis reports. NLM (Medline); 2021 [cited 2021 May 28]. p. 7. Available from: https://doi.org/10.1007/s11883-020-00901-4

14. Chen N, Zhou M, Dong X, Qu J, Gong F, Han Y, et al. Epidemiological and clinical characteristics of 99 cases of 2019 novel coronavirus pneumonia in Wuhan, China: a descriptive study. Lancet [Internet].2020Feb[cited2021Jul8];395(10223):507-13.Availablefrom:

https://www.sciencedirect.com/science/article/pii/S0140673620302117

15. Wang D, Hu B, Hu C, Zhu F, Liu X, Zhang J, et al. Clinical Characteristics of 138 Hospitalized Patients With 2019 Novel Coronavirus-Infected Pneumonia in Wuhan, China.

JAMA

[Internet]. 2020Mar17[cited2021Jul8];323(11):1061.Availablefrom: https://jamanetwork.com/journals/jama/article-abstract/2761044 16. Zheng C, Wang J, Guo H, Lu Z, Ma Y, Zhu Y, et al. Risk-adapted Treatment Strategy For COVID-19 Patients. Int J Infect Dis. 20201 May;94:74-7. 